\title{
Parametric Decoupling Control Strategy for a Class of Nonlinear Uncertain Systems via Observer-based Output Feedback
}

\author{
Qichun Zhang \\ School of Engineering and Sustainable Development \\ De Montfort University \\ Leicester, LE1 9BH, United Kingdom \\ Email: qichun.zhang@dmu.ac.uk
}

\author{
Xin Yin \\ Sino-British Joint Advanced Control System Technology Lab \\ University of Manchester \\ Mancheste, M13 9PL, United Kingdom \\ Email: leoxinyin@hotmail.com
}

\begin{abstract}
In this paper, the system decoupling problem has been investigated and a novel decoupling control strategy is presented for Lipschitz nonlinear uncertain multivariable systems. This control strategy consists of an explicit parametric state feedback controller and a linear state observer, where the free parameters of the controller can be adjusted to attenuate the coupling effects. In addition, the optimal parameters can be obtained using $H$ infinity norm based performance criterion. The convergence of the observer, the robust stabilization of the controller and closed-loop system are analyzed while the sufficient conditions are determined. Following the design procedure of the presented control strategy, an illustrative numerical example is given to demonstrate the effectiveness and correctness of the presented control strategy.
\end{abstract}

\section{INTRODUCTION}

Coupled or interactive phenomenon commonly exists in the complex industrial dynamic processes. How to design the decoupling controller for the MIMO system with strong coupling has gained widely attention since 1950s. Due to the coupling effects, the results for the SISO systems cannot be extended to the MIMO systems directly.

The decoupling problem described by state-space model was presented by B.S.Morgan[1] firstly. After years of development, P.L.Falb et al. [2], E.G.Gilbert et al.[3] and J.Descusse et al.[4] give the answers to this problem gradually. Corresponding developments also appear in the field of optimal decoupling control[5] and robust decoupling control [6]. All the control methods above focus on the linear deterministic models, however most of the practical processes are nonlinear systems, such as the Lipschitz nonlinear, thus the existing results have limitations from the view of implementation. To overcome these potential shortcoming forms the purpose of this paper.

To deal with the control design of the state space models with Lipschitz nonlinear and uncertain parameters, a plenty of results have been developed[7][8] while these nonlinear control laws are difficult to implement. In [9][10], the control structures are designed as the linear controllers with nonlinear compensators, however these nonlinear compensators process enormous online computation. Basically, it is significant to develop a simply control law for implementation for the complex dynamic systems to achieve decoupling.

Based upon the Lipschitz nonlinear uncertain multivariable model, the controller and linear observer can be designed and analyzed theoretically, while the nonlinear term can be considered as unmodeled dynamics which satisfies the Lipschitz condition. The sufficient conditions are given for the convergence of the observer, the robust stabilization of the controller and closed-loop system. Using this control structure, the design procedure is simplified and also the implement difficulty is reduced. Furthermore, the parameters of the controller and observer can be optimized following the parametric state feedback controller [11][12] and $\mathrm{H}$ infinity norm based performance index. Following the presented control strategy, the optimal output feedback control law has been obtained and the performance can be verified by the numerical simulation.

The rest of the paper is organized as follows. In Section 2, problem description is given including the model formulation and control objective. The optimal decoupling control strategy is developed while the convergence of the linear observer, the robust stabilization of the parametric state feedback linear controller and closed-loop nonlinear system are analyzed in Section 3. Moreover, the parameter optimization and design procedure are also discussed in this section. Section 4 presents the results of numerical simulation while the conclusions are drawn in Section 5.

\section{PROBLEM DESCRIPTION}

Suppose that the complex industrial dynamic process can be modelled by the following nonlinear uncertain multivariable systems.

$$
\begin{aligned}
\dot{x}(t)= & (A+\Delta A(t)) x(t)+(B+\Delta B(t)) u(t) \\
& +\phi(x(t), u(t)) \\
y(t)= & C x(t)
\end{aligned}
$$

where $x \in \mathbb{R}^{m}, u \in \mathbb{R}^{n}$ and $y \in \mathbb{R}^{n}$ are the system state vector, input vector and output vector, respectively. $m$ and $n$ are positive integers while system matrices $A, B C$ 
and parameter uncertainties $\Delta A(t), \Delta B(t)$ are of appropriate dimensions. The nonlinear term $\phi(x(t), u(t))$ is a vectorvalued nonlinear function.

Suppose that the investigated system model satisfies the following assumptions.

Assumption A1: $\phi(x(t), u(t))$ is Lipschitz with respect to the state $x$, uniformly in the control $u$, and there exists a real constant $\gamma_{c}>0$ such that

$$
\begin{aligned}
\left\|\phi\left(x_{1}, u_{1}\right)-\phi\left(x_{2}, u_{2}\right)\right\| & \leq \gamma_{c}\left\|x_{1}-x_{2}\right\| \\
\phi(0, u(t)) & =0
\end{aligned}
$$

Assumption A2: The pair $(A, B)$ is controllable and the pair $(A, C)$ is observable.

Assumption A3: The admissible parameter uncertainties are of the norm-bounded form

$$
\left[\begin{array}{cc}
\Delta A(t) & \Delta B(t)
\end{array}\right]=M\left[\begin{array}{ll}
\Xi_{1}(t) N_{1} & \Xi_{2}(t) N_{2}
\end{array}\right]
$$

In Eq. (3), $M, N_{1}$ and $N_{2}$ denote the structure of the uncertainties are known real constant matrices with proper dimensions, $\Xi_{1}(t)$ and $\Xi_{2}(t)$ are unknown time-varying matrices which respectively meet the following conditions.

$$
\Xi_{1}^{T}(t) \Xi_{1}(t) \leq I, \Xi_{2}^{T}(t) \Xi_{2}(t) \leq I
$$

Remark 1: All the assumptions mentioned above are common in nonlinear control. In particular, the parameter uncertainty structure in Eq. (3) has been widely used in the problem of robust stabilization of uncertain systems[13]. Moreover, it can represent parameter uncertainty in many physical cases.

The control objective is to develop a new control strategy so that the closed-loop system remains robust stabilization and the optimal control law should be designed to decouple the system outputs $y$.

\section{Control Strategy}

\section{A. State feedback design}

The linear state feedback controller can be determined by the nominal linear model and the control law is described by

$$
u(t)=K x(t)
$$

where the gain matrix $K$ can be obtained by parametric design[12] while

$$
\begin{aligned}
K & =\left[W_{1} f_{1}, \ldots, W_{m} f_{m}\right] \\
& \times\left[\left(\lambda_{1}^{*} I-A_{1}\right)^{-1} B_{1} f_{1}, \ldots,\left(\lambda_{m}^{*} I-A_{m}\right)^{-1} B_{m} f_{m}\right]^{-1}
\end{aligned}
$$

where modified parameter vectors and closed-loop eigenvalues are denoted by $f_{1}, \ldots, f_{m}$ and $\lambda_{1}^{*}, \ldots, \lambda_{m}^{*}$ which can be considered as free parameters. In the case of a common openloop and closed-loop eigenvalue, the gain matrix $K$ can be determined by the following equations.

$$
\begin{aligned}
A_{j} & =A+v_{j}^{0} w_{j}^{0^{T}} \\
W_{j} & =I-\frac{e_{i}{w_{j}^{0}}^{T} B}{w_{j}^{0^{T}} b_{i}} \\
B_{j} & =B W_{j}+v_{j}^{0} e_{i}^{T}, j=1, \ldots, m
\end{aligned}
$$

where $v_{j}^{0}$ and $w_{j}^{0}$ denote the open-loop eigenvectors and eigenrows of $A . b_{i}$ is the $i$-th column of $B . e_{i}$ is a unit vector while the $i$-th element is 1 . In the other case, no common eigenvalue results in $w_{j}^{0 T} b_{i}=0$ which leads to

$$
\begin{aligned}
A_{j} & =A \\
W_{j} & =I \\
B_{j} & =B, j=1, \ldots, m
\end{aligned}
$$

Substituting control law (5) into the system model (1) yields the closed-loop system:

$$
\dot{x}(t)=\left(A_{c}+\Delta A_{c}(t)\right) x(t)+\phi(x(t), u(t))
$$

where $A_{c}=A+B K, \Delta A_{c}(t)=\Delta A(t)+\Delta B(t) K$. Thus the following lemma can be proposed.

Lemma 2: For the nonlinear uncertain multi-variable system given by (1), with the assumptions A1, A2, A3 and with the control law given by (5), then there exist three positive constants $\varepsilon_{1}, \varepsilon_{2}, \varepsilon_{3}$, so that the equilibrium $x(t)=0$ is stabilized if the following matrix inequality has a positivedefinite solution $P=P^{T}>0$.

$$
\begin{array}{r}
A_{c}^{T} P+P A_{c}+\varepsilon_{1} N_{1}^{T} N_{1}+\left(\varepsilon_{1}^{-1}+\varepsilon_{2}^{-1}\right) P M M^{T} P \\
+\varepsilon_{2} K^{T} N_{2}^{T} N_{2} K+\varepsilon_{3} P^{T} P+\varepsilon_{3}^{-1} \gamma^{2} I<0
\end{array}
$$

Proof: Consider the Lyapunov function candidate as

$$
V_{c}(x)=x^{T}(t) P x(t), P=P^{T}>0
$$

The time derivative of $V_{c}(x)$ along the trajectories of (9) is

$$
\begin{aligned}
& \dot{V}_{c}(x)=x^{T}(t) A_{c}^{T} P x(t)+x^{T}(t) P A_{c} x(t) \\
& \quad+x^{T}(t) P \phi(x(t), u(t))+\phi^{T}(x(t), u(t)) P x(t) \\
& \quad+x^{T}(t) \Delta A^{T}(t) P x(t)+x^{T}(t) P \Delta A(t) x(t) \\
& \quad+x^{T}(t) K^{T} \Delta B^{T}(t) P x(t)+x^{T}(t) P \Delta B(t) K x(t)
\end{aligned}
$$

Let $\varepsilon_{1}, \varepsilon_{2}$ and $\varepsilon_{3}$ be positive constants. Thus the following matrix inequalities hold using the Lemma in [14].

$$
\begin{aligned}
& x^{T}(t) \Delta A^{T}(t) P x(t)+x^{T}(t) P \Delta A(t) x(t) \\
& \quad=x^{T}(t)\left(M \Xi_{1} N_{1}\right)^{T} P x(t)+x^{T}(t) p M \Xi_{1} N_{1} x(t) \\
& \quad \leq x^{T}(t)\left(\varepsilon_{1} N_{1}^{T} N_{1}+\varepsilon_{1}^{-1} P M M^{T} P\right) x(t)
\end{aligned}
$$

$$
\begin{aligned}
& x^{T}(t) K^{T} \Delta B^{T}(t) P x(t)+x^{T}(t) P \Delta B(t) K x(t) \\
& =x^{T}(t) K^{T}\left(M \Xi_{2} N_{2}\right)^{T} P x(t)+x^{T}(t) P M \Xi_{2} N_{2} K x(t) \\
& \leq x^{T}(t)\left(\varepsilon_{2} K^{T} N_{2}^{T} N_{2} K+\varepsilon_{2}^{-1} P M M^{T} P\right) x(t)
\end{aligned}
$$

$$
\begin{aligned}
& x^{T}(t) P \phi(x(t), u(t))+\phi^{T}(x(t), u(t)) P x(t) \\
& \leq \varepsilon_{3} x^{T}(t) P^{T} P x(t)+\varepsilon_{3}^{-1} \phi^{T}(x(t), u(t)) \phi(x(t), u(t)) \\
& \leq x^{T}(t)\left(\varepsilon_{3} P^{T} P+\varepsilon_{3}^{-1} \gamma^{2} I\right) x(t)
\end{aligned}
$$


Substituting these inequalities into the derivative of $V_{c}(x)$ with $\mathrm{A} 1$, we have

$$
\begin{aligned}
\dot{V}_{c}(x) & \leq x^{T}(t)\left(A_{c}^{T} P+P A_{c}\right) x(t) \\
& +x^{T}(t)\left(\varepsilon_{1} N_{1}^{T} N_{1}+\varepsilon_{1}^{-1} P M M^{T} P\right) x(t) \\
& +x^{T}(t)\left(\varepsilon_{2} K^{T} N_{2}^{T} N_{2} K+\varepsilon_{2}^{-1} P M M^{T} P\right) x(t) \\
& +x^{T}(t)\left(\varepsilon_{3} P^{T} P+\varepsilon_{3}^{-1} \gamma^{2} I\right) x(t)
\end{aligned}
$$

Since $\dot{V}_{c}(x)<0$, the proof of lemma 1 is completed.

\section{B. Observer design}

Using the linear observer to estimate the states of the model (1), the linear observer can be designed based on the nominal linear model.

$$
\dot{\hat{x}}(t)=(A-L C) \hat{x}(t)+L y(t)+B u(t)
$$

where the estimated state vector can be denoted by $\hat{x}$ and $L$ is the gain matrix of this observer.

Introducing the error of the estimation by

$$
e(t)=x(t)-\hat{x}(t)
$$

and substituting the Eq. (17)-(18) to system model (1). The closed-loop model can be described by

$$
\dot{e}(t)=A_{o} e(t)+\Delta A_{c}(t) x(t)+\phi(x(t), u(t))
$$

where $A_{o}=A-L C$. Similar to Lemma 2, Lemma 3 is given as follows.

Lemma 3: For the nonlinear uncertain multi-variable system given by (1), with the assumptions A1, A2, A3 and with the linear observer given by (17), then there exists three positive constants $\varepsilon_{1}, \varepsilon_{2}, \varepsilon_{3}$, so that the estimation error $e(t)$ converges to zero if the following matrix inequalities have a positivedefinite solution $P=P^{T}>0$.

$$
\begin{gathered}
A_{o} P+P A_{o}+\varepsilon_{3} P^{T} P<0 \\
\varepsilon_{1} N_{1}^{T} N_{1}+\left(\varepsilon_{1}^{-1}+\varepsilon_{2}^{-1}\right) P M M^{T} P \\
+\varepsilon_{2} K^{T} N_{2}^{T} N_{2} K+\varepsilon_{3}^{-1} \gamma^{2} I<0
\end{gathered}
$$

Proof: Consider the Lyapunov function candidate as

$$
V_{o}(e)=e^{T}(t) P e(t), P=P^{T}>0
$$
is

The time derivative of $V_{o}(e)$ along the trajectories of (18)

$$
\begin{aligned}
& \dot{V}_{o}(e)=e^{T}(t)\left(A_{o} P+P A_{o}\right) e(t) \\
& \quad+e^{T}(t) P \phi(x(t), u(t))+x^{T}(t) \Delta A^{T}(t) P x(t) \\
& \quad+\phi(x(t), u(t))^{T} P e(t)+x^{T}(t) P \Delta A(t) x(t) \\
& \quad+x^{T}(t) K^{T} \Delta B^{T}(t) P x(t)+x^{T}(t) P \Delta B(t) K x(t)
\end{aligned}
$$

Similar to the proof of Lemma 2, we have

$$
\begin{aligned}
\dot{V}_{o}(e) & \leq e^{T}(t)\left(A_{o} P+P A_{o}\right) e(t) \\
& +x^{T}(t)\left(\varepsilon_{1} N_{1}^{T} N_{1}+\varepsilon_{1}^{-1} P M M^{T} P\right) x(t) \\
& +x^{T}(t)\left(\varepsilon_{2} K^{T} N_{2}^{T} N_{2} K+\varepsilon_{2}^{-1} P M M^{T} P\right) x(t) \\
& +\varepsilon_{3} e^{T}(t) P^{T} P e(t)+\varepsilon_{3}^{-1} \gamma^{2} x^{T}(t) x(t)
\end{aligned}
$$

\section{Output feedback design}

Combining the parametric state feedback controller and the observer which mentioned above, the output feedback controller should be designed for the system (1).

$$
u(t)=K \hat{x}(t)
$$

which leads to the closed-loop dynamic as follows.

$$
\begin{array}{r}
\dot{x}(t)=A_{c} x(t)+\Delta A_{c}(t) x(t)+\phi(x(t), u(t)) \\
-(B+\Delta B(t)) K e(t)
\end{array}
$$

Then the stability of the closed-loop control design can be guaranteed by the following theorem

Theorem 4: For the nonlinear uncertain multi-variable system given by (1), with the assumptions A1, A2, A3 and with the control law given by (25) using the observer (17), then there exists a set of positive constants $\varepsilon_{i}, i=1, \ldots, 8$, so that the equilibrium $x(t)=0$ is stabilized if the following matrix inequalities have positive-definite solution $P_{1}=P_{1}^{T}>$ $0, P_{2}=P_{2}^{T}>0$.

$$
\begin{aligned}
\varepsilon_{4} K^{T} B^{T} B K+\varepsilon_{5} K^{T} N_{2}^{T} N_{2} K+ & A_{o} P_{2}+P_{2} A_{o} \\
& +\varepsilon_{8} P_{2}^{T} P_{2}<0
\end{aligned}
$$

$$
\begin{aligned}
& A_{c}^{T} P_{1}+P_{1} A_{c}+\left(\varepsilon_{1}^{-1}+\varepsilon_{2}^{-1}+\varepsilon_{4}^{-1}+\varepsilon_{5}^{-1}\right) P_{1} M M^{T} P \\
& +\left(\varepsilon_{6}^{-1}+\varepsilon_{7}^{-1}\right) P_{2} M M^{T} P_{2}+\left(\varepsilon_{2}+\varepsilon_{7}\right) K^{T} N_{2}^{T} N_{2} K \\
& +\varepsilon_{3} P_{1}^{T} P_{1}+\left(\varepsilon_{3}^{-1}+\varepsilon_{8}^{-1}\right) \gamma^{2} I_{1}+\left(\varepsilon_{1}+\varepsilon_{6}\right) N_{1}^{T} N_{1}<0
\end{aligned}
$$

Proof: Consider the Lyapunov function candidate as

$$
V(x(t), e(t))=x^{T}(t) P_{1} x(t)+e^{T}(t) P_{2} e(t)
$$

The time derivative of $V(x(t), e(t))$ along the trajectories of (26) is

$$
\begin{aligned}
& \dot{V}(x(t), e(t))=x^{T} A_{c}^{T} P x+x^{T} P A_{c} x+\phi^{T}(x, u) P x \\
& +x^{T} P \phi(x, u)+x^{T} \Delta A^{T} P x+x^{T} P \Delta A x \\
& +x^{T} K^{T} \Delta B^{T} P x+x^{T} P \Delta B K x-e^{T} K^{T} B^{T} P x \\
& -x^{T} P B K e-e^{T} K^{T} \Delta B^{T} P x-x^{T} P \Delta B K e \\
& +e^{T}(t)\left(A_{o} P+P A_{o}\right) e(t)+e^{T}(t) P \phi(x(t), u(t)) \\
& +\phi(x(t), u(t))^{T} P e(t)+x^{T}(t) \Delta A^{T}(t) P x(t) \\
& +x^{T}(t) P \Delta A(t) x(t)+x^{T}(t) K^{T} \Delta B^{T}(t) P x(t) \\
& +x^{T}(t) P \Delta B(t) K x(t)
\end{aligned}
$$

Let $\varepsilon_{4}$ and $\varepsilon_{5}$ be positive constants. Thus the following matrix inequalities hold using the Lemma in [14].

$$
\begin{aligned}
& -e^{T}(t) K^{T} B^{T} P x(t)-x^{T}(t) P B K e(t) \\
\leq & \varepsilon_{4} e^{T}(t) K^{T} B^{T} B K e(t)+\varepsilon_{4}^{-1} x^{T}(t) P M M^{T} P x(t) \\
& \quad-e^{T}(t) K^{T} \Delta B^{T}(t) P x(t)-x^{T}(t) P \Delta B(t) K e(t) \\
\leq & \varepsilon_{5} e^{T}(t) K^{T} N_{2}^{T} N_{2} K e(t)+\varepsilon_{5}^{-1} x^{T}(t) P M M^{T} P x(t)
\end{aligned}
$$

which ends the proof 
Substituting these inequalities into the derivative of $V(x(t), e(t))$ and using Lemma 3, we have

$$
\begin{aligned}
\dot{V} & \leq x^{T}\left[A_{c}^{T} P_{1}+P_{1} A_{c}+\left(\varepsilon_{1}+\varepsilon_{6}\right) N_{1}^{T} N_{1}\right. \\
& +\left(\varepsilon_{2}+\varepsilon_{7}\right) K^{T} N_{2}^{T} N_{2} K+\varepsilon_{3} P_{1}^{T} P_{1}+\left(\varepsilon_{3}^{-1}+\varepsilon_{8}^{-1}\right) \gamma^{2} I \\
& +\left(\varepsilon_{1}^{-1}+\varepsilon_{2}^{-1}+\varepsilon_{4}^{-1}+\varepsilon_{5}^{-1}\right) P_{1} M M^{T} P_{1} \\
& \left.+\left(\varepsilon_{6}^{-1}+\varepsilon_{7}^{-1}\right) P_{2} M M^{T} P_{2}\right] x \\
& +e^{T}\left(\varepsilon_{4} K^{T} B^{T} B K+\varepsilon_{5} K^{T} N_{2}^{T} N_{2} K\right. \\
& \left.+A_{o} P_{2}+P_{2} A_{o}+\varepsilon_{8} P_{2}^{T} P_{2}\right) e
\end{aligned}
$$

which leads to the conditions and the proof has been completed.

\section{Parametric optimisation}

To deal with the coupling effects of the MIMO system, the free parameters of the controller should be optimized. Substituting the feedback gain matrix (6) and the control law (25) to the nominal linear model which is used to design the controller, the linear closed-loop model can be obtained as follows.

$$
\begin{aligned}
& \dot{x}(t)=A_{c} x(t) \\
& y(t)=C x(t)
\end{aligned}
$$

The transfer function matrix of this state space model (34) can be obtained by

$$
G\left(s, \lambda_{i}^{*}, f_{i}\right)=C\left(s I-A_{c}\right)^{-1} B
$$

where the elements of the matrix are transfer functions which can be changed by turning the free parameters of the controller. Furthermore, the Eq. (35) can be expressed as another form by

$$
G\left(s, \lambda_{i}^{*}, f_{i}\right)=\bar{G}\left(s, \lambda_{i}^{*}, f_{i}\right)+\overline{\bar{G}}\left(s, \lambda_{i}^{*}, f_{i}\right)
$$

where the matrix $\bar{G}$ denotes the diagonal matrix and the diagonal elements of matrix $\overline{\bar{G}}$ are zero.

The coupling effects should be attenuate if the $\mathrm{H}$ infinity norm of the matrix is close to zero, and also the norm of the matrix should be close to one, in order to meet the control objective. Therefore, two performance indexes can be proposed as follows.

$$
\begin{aligned}
& J_{1}\left(\lambda_{i}^{*}, f_{i}\right)=\min \sum_{j=1}^{m} \sum_{i=1}^{m}\left\|G_{i j}^{*}\left(s, \lambda_{i}^{*}, f_{i}\right)\right\|_{H_{\infty}}, j \neq i \\
& J_{2}\left(\lambda_{i}^{*}, f_{i}\right)=\min \sum_{j=1}^{m} \sum_{i=1}^{m}\left\|1-G_{i j}^{*}\left(s, \lambda_{i}^{*}, f_{i}\right)\right\|_{H_{\infty}}, j=i
\end{aligned}
$$

Comparing (1) and (34), the nonlinear dynamic with unmatched time-varying uncertain parameters can be considered as random disturbances which will also affect the performance of the decoupling design. The probabilistic decoupling[15] should be considered as a compensation performance index.

$$
J_{3}=\min \left\|\gamma_{J}\left(y_{k}\right)-\prod_{i=0}^{n} \gamma_{i}\left(y_{i, k}\right)\right\|_{H_{\infty}}
$$

where $\gamma_{J}$ and $\gamma_{i}$ denote the joint probability density function and the marginal probability density function for each system output $y_{i}$, respectively. Moreover these probability density functions in Eq. (39) can be estimated by the kernel density estimation [16].

Therefore, the complete performance criterion can be given as follows.

$$
J=R_{1} J_{1}\left(\lambda_{i}^{*}, f_{i}\right)+R_{2} J_{2}\left(\lambda_{i}^{*}, f_{i}\right)+R_{3} J_{3}
$$

where real positive $R_{1}, R_{2}$ and $R_{3}$ denote the performance weights.

Then the optimal free parameter $f_{i}$ can be obtained by gradient descent once the eigenvalues $\lambda_{i}^{*}$ are pre-specified.

$$
f_{i, j+1}=f_{i, j}+\left.\mu \frac{d J}{d f_{i}}\right|_{f_{i}=f_{i, j}} i=1, \ldots, m
$$

where $j$ denotes the optimisation searching iteration index. $\mu$ stands for the pre-specified step. Note that the free parametric optimization would not affect the stability of the closed-loop system design.

Remark 5: The feedback gain matrix of the observer can be obtained using proposed optimization approach in this section based on the dual principle.

Remark 6: The optimization operation can also be replaced by multi-objective optimization algorithms then the weights can be neglected.

Remark 7: Only a few elements of the parameter vectors $f_{i}$ affect the control performance directly. Therefore, in order to determine the free parameters quickly, trial and error method can be used, and the performance indexes can verify the manually selected parameters simply.

\section{E. Design procedure}

The procedure of the proposed control strategy is summarized as follows:

Step1 Setup the initial free parameters of the controller.

Step2 Transfer the closed-loop model to the transfer function matrix and develop the expressions of the performance indexes.

Step3 Use the numerical approach to optimize the performance indexes, by computing the $\mathrm{H}$ infinity norm and multiobjective optimization, and the optimal parameters are obtained.

Step4 Compute the feedback gain matrix of the control law, and verify it by the conditions of Lemma 2 to guarantee robust stability of the system, if the conditions hold, then go to next step, otherwise, return to step 1.

Step5 Obtain the feedback gain matrix of the observer by dual principle and verify it by Lemma 3 .

Step6 Verify the optimal parameters by the theorem 4, and if the conditions can be satisfied, then complete the procedure, otherwise, return to step 1 . 


\section{A Numerical Simulation}

In order to illustrate the effectiveness of decoupling control strategy proposed in this paper, a numerical simulation has been carried out.

Consider the parameters of the Lipschitz nonlinear uncertain multivariable systems (1) as follows.

$$
\begin{gathered}
A=\left[\begin{array}{ccc}
2 & 0 & -1 \\
1 & -1 & -1 \\
1 & -1 & -2
\end{array}\right], B=\left[\begin{array}{ll}
1 & 0 \\
0 & 1 \\
0 & 1
\end{array}\right] \\
C=\left[\begin{array}{lll}
1 & 1 & 0 \\
0 & 0 & 1
\end{array}\right], M=\left[\begin{array}{ccc}
0.1 & 0 & 0.1 \\
0 & 0.2 & 0 \\
0.1 & 0 & 0.1
\end{array}\right] \\
\Xi_{1}(t)=\Xi_{2}(t)=\sin t\left[\begin{array}{ccc}
1 & 0 & 0 \\
0 & 1 & 0 \\
0 & 0 & 1
\end{array}\right] \\
N_{1}=\left[\begin{array}{ccc}
0.1 & 0.1 & 0 \\
0 & 0 & 0.1 \\
0 & 0.1 & 0
\end{array}\right], N_{2}=\left[\begin{array}{cc}
0 \\
0 \\
0.1 & 0.1
\end{array}\right] \\
\phi(x(t), u(t))=\left[\begin{array}{c}
0.1 \sin x_{1}+0.1 \sin x_{2} \\
0.1 \sin x_{3} \\
0.1 \sin x_{1}+0.1 \sin x_{3}
\end{array}\right]
\end{gathered}
$$

Pre-selecting the free parameters $\lambda_{i}^{*}$ and setting the initial values of $f_{i}$, the optimal parameters can be obtained by computing the performance indexes

$$
\begin{aligned}
& \lambda_{1}^{*}=-1, \lambda_{2}^{*}=-2, \lambda_{3}^{*}=-3, f_{1}^{*}=\left(\begin{array}{cc}
3 & 1
\end{array}\right)^{T} \\
& f_{2}^{*}=\left(\begin{array}{ll}
9.98 & -1.1
\end{array}\right)^{T}, f_{3}^{*}=\left(\begin{array}{ll}
0 & 0.97
\end{array}\right)^{T}
\end{aligned}
$$

Using the Eq.(6) and the dual principle, both the feedback gain matrix of the controller and the observer can be developed.

$$
\begin{aligned}
& K^{*}=\left[\begin{array}{rrr}
-4.5231 & -2.8615 & 2.4308 \\
-1.5077 & -1.9538 & 1.4769
\end{array}\right] \\
& L=\left[\begin{array}{cc}
27.4089 & -6.9170 \\
-9.2883 & 2.3958 \\
4.4179 & 4.8795
\end{array}\right]
\end{aligned}
$$

Substituting the parameters, the closed-loop dynamic can be simulated with the initial states $x_{1}=x_{2}=x_{3}=0.5$. Then Fig. (1-3) show the control performance, the estimated states, and the estimation errors, respectively. From the results, the controller and observer can meet the control objective for the Lipschitz nonlinear uncertain multivariable system (1) while the procedure of designing is simply and easy to implement.

In order to illustrate the decoupling performance, a disturbance sine wave, Fig. (4), is introduced to the closed-loop dynamic to affect the control signals, and the amplitude, frequency of this wave are $0.1,2$, respectively. Another feedback gain matrix of the controller $K$ is selected to compare with the optimal parameters.

$$
K=\left[\begin{array}{ccc}
-9.2609 & -5.9565 & 5.1739 \\
19.0870 & 16.6522 & -12.3913
\end{array}\right]
$$

The control performance with the different feedback gain matrixes can be showed by Fig. (4) and Fig. (5). By analyzing

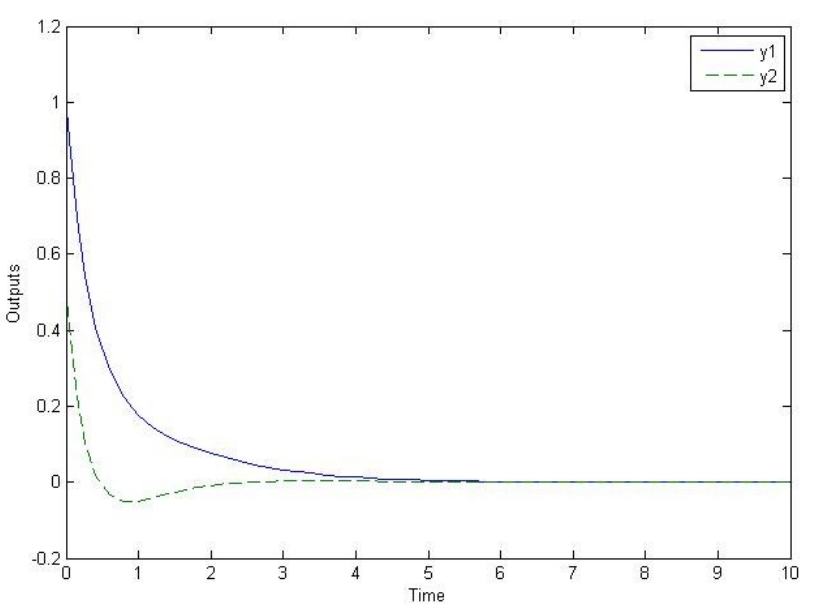

Fig. 1. The outputs of the closed-loop system.

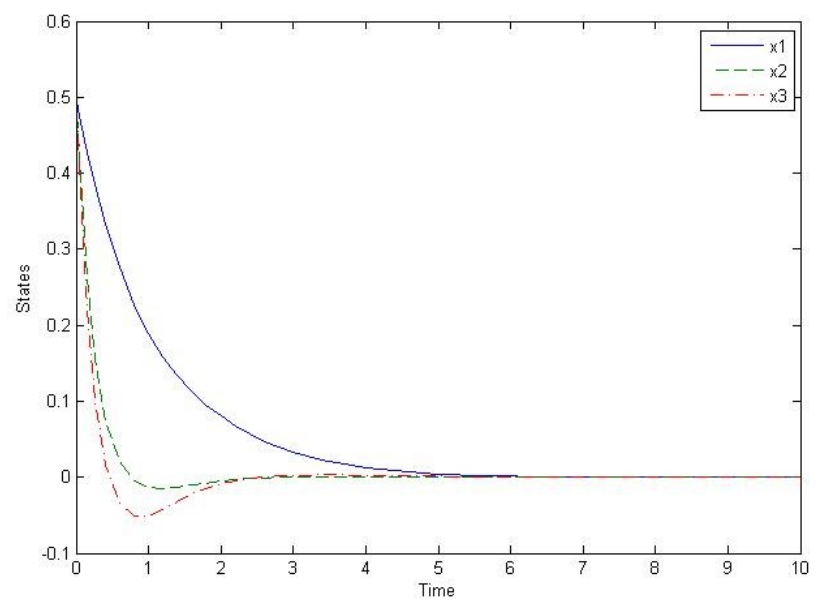

Fig. 2. The estimated states.

the results of the simulation, the decoupling performance with the optimal parameters is better and the coupling affects have been attenuated. When the system inputs periodic fluctuate with the given sine wave disturbance, the coupling effects of the system outputs still exist using feedback gain matrix $K$ showing by Fig. (4), while the outputs $y_{1}, y_{2}$ seem almost unaffected by the non-diagonal control inputs $u_{1}, u_{2}$, and also the performance of the diagonal outputs become better.

\section{CONCLUSION}

The problem of decoupling for a class of nonlinear uncertain system has been solved using the parametric control strategy. Combining the $\mathrm{H}$ infinity norm and parametric optimization, the optimal parameters for decoupling design have been obtained by optimizing proposed performance criterion. And also stability analysis in this paper is given to guarantee the robustness, stabilization and convergence of the system. From the results of the numerical simulation, the effectiveness of the 


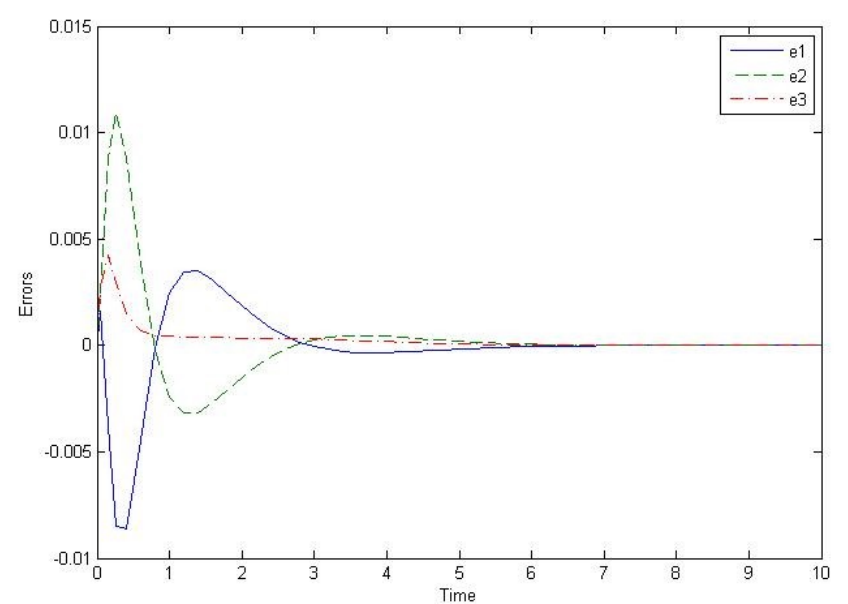

Fig. 3. The estimation errors of the observer.
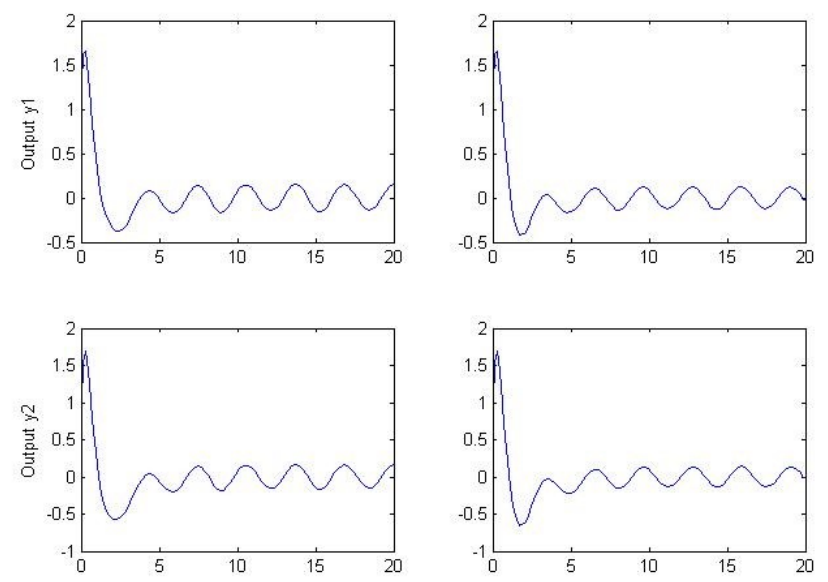

Fig. 4. The decomposed outputs with the pulse using $K$.

proposed decoupling control strategy has been verified. The benefit of the proposed control strategy is that the controller is easy to design and implement when the nonlinear term is unknown with parameter uncertainties.

Due to the nonlinear of the performance indexes, the parametric optimization is difficult to develop the globally optimal solution, and the locally optimal solution is associated with the initial value. Therefore, more intelligent optimization methods will be used to optimize the parameters as the future works.

\section{ACKNOWLEDGMENT}

The authors would like to thank Prof Hong Wang for his helpful suggestions on various technical issues in this paper, and the anonymous revierwers for their valueable comments.

\section{REFERENCES}

[1] B. Morgan, "The synthesis of linear multivariable systems by statevariable feedback," IEEE Transactions on Automatic Control, vol. 9, no. 4, pp. 405-411, 1964.
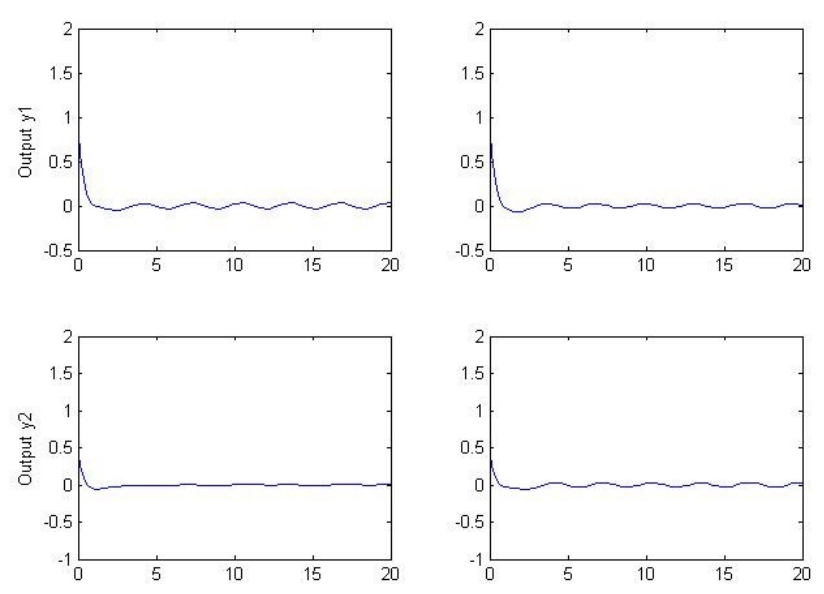

Fig. 5. The decomposed outputs with the pulse using $K^{*}$.

[2] P. Falb and W. Wolovich, "Decoupling in the design and synthesis of multivariable control systems," IEEE transactions on Automatic Control, vol. 12, no. 6, pp. 651-659, 1967.

[3] E. Gilbert and J. Pivnichny, "A computer program for the synthesis of decoupled multivariable feedback systems," IEEE Transactions on Automatic Control, vol. 14, no. 6, pp. 652-659, 1969.

[4] J. Descusse, J. Lafay, and M. Malabre, "Solution to morgan's problem," IEEE Transactions on Automatic Control, vol. 33, no. 8, pp. 732-739, 1988.

[5] K. Park, G.-H. Choi, and T.-Y. Kuc, "Wiener-hopf design of the optimal decoupling control system with state-space formulas," Automatica, vol. 38, no. 2, pp. 319-326, 2002.

[6] G. Conte and A. M. Perdon, "Robust disturbance decoupling problem for parameter dependent families of linear systems," Automatica, vol. 29, no. 2, pp. 475-478, 1993.

[7] Z. Wang, B. Huang, and H. Unbehauen, "Robust reliable control for a class of uncertain nonlinear state-delayed systems," Automatica, vol. 35, no. 5, pp. 955-963, 1999.

[8] T. Shen and K. Tamura, "Robust h/sub/spl infin//control of uncertain nonlinear system via state feedback," IEEE Transactions on Automatic Control, vol. 40, no. 4, pp. 766-768, 1995.

[9] Y. Zhang, T. Chai, and H. Wang, "A nonlinear control method based on anfis and multiple models for a class of siso nonlinear systems and its application," IEEE Transactions on Neural Networks, vol. 22, no. 11, pp. 1783-1795, 2011.

[10] T. Chai, Y. Zhang, H. Wang, C.-Y. Su, and J. Sun, "Data-based virtual unmodeled dynamics driven multivariable nonlinear adaptive switching control," IEEE Transactions on Neural Networks, vol. 22, no. 12, pp. 2154-2172, 2011.

[11] G. Roppenecker, "On parametric state feedback design," International Journal of Control, vol. 43, no. 3, pp. 793-804, 1986.

[12] Q. Zhang, Z. Wang, and H. Wang, "Parametric covariance assignment using a reduced-order closed-form covariance model," Systems Science \& Control Engineering, vol. 4, no. 1, pp. 78-86, 2016.

[13] P. P. Khargonekar, I. R. Petersen, and K. Zhou, "Robust stabilization of uncertain linear systems: quadratic stabilizability and h/sup infinity/control theory," IEEE Transactions on Automatic Control, vol. 35, no. 3, pp. 356-361, 1990.

[14] I. R. Petersen, "A stabilization algorithm for a class of uncertain linear systems," Systems \& Control Letters, vol. 8, no. 4, pp. 351-357, 1987.

[15] Q. Zhang, J. Zhou, H. Wang, and T. Chai, "Minimized coupling in probability sense for a class of multivariate dynamic stochastic control systems," in Decision and Control (CDC), 2015 IEEE 54th Annual Conference on. IEEE, 2015, pp. 1846-1851.

[16] Q. Zhang and A. Wang, "Decoupling control in statistical sense: minimised mutual information algorithm," International Journal of Advanced Mechatronic Systems, vol. 7, no. 2, pp. 61-70, 2016. 